\title{
Supplementation of DHA but not DHA with arachidonic acid during pregnancy and lactation influences general movement quality in 12-week-old term infants
}

\author{
Saskia A. van Goor ${ }^{1 *}$, D. A. Janneke Dijck-Brouwer ${ }^{1}$, Bennard Doornbos ${ }^{2}$, Jan Jaap H. M. Erwich ${ }^{3}$, \\ Anne Schaafsma ${ }^{4}$, Frits A. J. Muskiet ${ }^{1}$ and Mijna Hadders-Algra ${ }^{5}$ \\ ${ }^{1}$ Laboratory Medicine, University Medical Center Groningen, Internal postal code EA22, PO Box 30.001, 9700 RB Groningen, \\ The Netherlands \\ ${ }^{2}$ Department of Psychiatry, University Medical Center Groningen, Groningen, The Netherlands \\ ${ }^{3}$ Obstetrics and Gynaecology, University Medical Center Groningen, Groningen, The Netherlands \\ ${ }^{4}$ FrieslandCampina, Leeuwarden, The Netherlands \\ ${ }^{5}$ Department of Paediatrics, University Medical Center Groningen, Groningen, The Netherlands \\ (Received 27 January 2009 - Revised 14 July 2009 - Accepted 15 July 2009 - First published online 25 August 2009)
}

\begin{abstract}
DHA and arachidonic acid (AA) are important for neurodevelopment. A traditional neonatal neurological examination and the evaluation of general movement quality are sensitive techniques for assessing neurodevelopment in young infants. Mildly abnormal general movements at 3 months have been associated with a non-optimal current brain condition. We investigated whether supplementation of DHA during pregnancy and lactation influences the infant's brain development and whether additional AA modulates this effect. Healthy women were randomly assigned to DHA (220 mg/d, $n$ 42), DHA + AA (220 mg each/d, $n$ 41) or control ( $n$ 36), from about week 17 (range 14-20 weeks) of pregnancy until 12 weeks postpartum. The control and the DHA + AA groups had approximately comparable dietary DHA/AA ratios. The standardised neonatal neurological examination was carried out at 2 weeks. General movement quality was assessed at 2 and 12 weeks. Neither DHA alone nor DHA + AA influenced outcomes in the traditional examination. General movement quality of infants in the DHA group was lower than that of infants in the other two groups, especially at 12 weeks: $61 \%$ of the infants in the DHA group showed mildly abnormal general movements compared with $31 \%$ in the control group $(P=0.008)$ and $34 \%$ in the DHA + AA group $(P=0 \cdot 015)$. We conclude that general movement quality at 12 weeks is sensitive to the maternal dietary DHA/AA balance.
\end{abstract}

DHA: Arachidonic acid: Supplementation: Pregnancy: General movements: Infant brain development

The long-chain PUFA (LCP) DHA (22:6n-3) and arachidonic acid (AA; 20:4n-6) are structural components of membrane phospholipids, modulators of gene expression and precursors of eicosanoids, resolvins and (neuro)protectins ${ }^{(1)}$. DHA and EPA $(20: 5 n-3)$ are mainly derived from fish, while meat, poultry and eggs are the most important sources of dietary AA. Both DHA and AA are considered important for brain development.

Animal studies and to a lesser extent human trials with $n-3$ LCP in pregnant women and newborns showed that neonatal brain DHA is positively related to cognitive and behavioural performance and that small differences in brain DHA content may result in subtle effects ${ }^{(2)}$. However, although many studies addressed the influence of DHA or fish oil supplementation on several aspects of brain development, the results remain inconclusive at best ${ }^{(2)}$. The high accretion rate of $\mathrm{AA}$ in the fetal brain, especially in early pregnancy ${ }^{(3)}$, and the positive association between prenatal AA status and neurodevelopment ${ }^{(4)}$ suggest that not only adequate fetal and maternal DHA, but also adequate fetal and maternal AA status, is important. In addition, maternal LCP status is known to decline during pregnancy ${ }^{(5)}$. As far as we know, no studies have been performed on the effects of maternal supplemental AA or DHA + AA during pregnancy and lactation on neurodevelopment in human subjects.

Since the effects of supplemental LCP during pregnancy and lactation are expected to be subtle, sensitive techniques are needed to evaluate their effects on brain development. In the present study, we applied a traditional neurological examination $^{(6)}$, which yields a neurological classification and a neurological optimality score, while we also assessed the quality of general movements ${ }^{(7)}$. The outcomes of the two techniques have previously been shown to be sensitive to changes in LCP status ${ }^{(8,9)}$. Both techniques assess the integrity of the young nervous system, which - in contrast to the adult nervous system - is organised in a relatively generalised

Abbreviations: AA, arachidonic acid; ALA, $\alpha$-linolenic acid; LA, linoleic acid; LCP, long-chain PUFA.

* Corresponding author: Saskia A. van Goor, fax +31 50361 2290, email saskiavangoor@yahoo.com 
manner. The developmental difference in organisation between the infant and adult brain is, for instance, the reason why even severe impairments such as cerebral palsy can only be initially diagnosed from 18 months on. The traditional neurological examination summarises the spinal cord, brainstem and cortico-subcortical function to an equal extent, whereas the assessment of general movements especially provides information on the integrity of corticalsubcortical circuitries ${ }^{(10)}$.

We investigated whether maternal DHA supplementation influences early neurological outcomes and whether additional AA modulates the effect of DHA.

\section{Subjects and methods}

\section{Participants}

The present study was part of a double-blind placebocontrolled randomised trial in which we explored the influence of DHA with or without AA during pregnancy and lactation on infant neurodevelopment, maternal mood ${ }^{(11)}$ and cognition, and on the fatty acid composition of $\operatorname{milk}^{(12)}$. Apparently healthy pregnant women were recruited by midwives or gynaecologists in and about the city of Groningen in The Netherlands. The inclusion of participants took place from December 2004 until December 2006. We included women with a first or second low-risk singleton pregnancy and excluded women with vegetarian or vegan diets and women with diabetes mellitus. Women were enrolled between the 14 th and 20th weeks of pregnancy, with the majority $(80 \%)$ being enrolled between 15.6 and 17.4 weeks postmenstrual age (mean 16.5 weeks). Stop criteria for participation were delivery before 37 or after 42 weeks and any maternal or neonatal complications. At enrolment, the women were randomised to three groups using block randomisation.

We included 183 women in the trial, 58 of whom dropped out before week 36 of pregnancy due to a lack of motivation to complete the questionnaires on a regular basis and take supplements daily ( $n 23$ in the control group, $n 20$ in the DHA group and $n 15$ in the DHA + AA group). In the control group, three mother-infant pairs were excluded because of preterm delivery. In the DHA group, one woman was excluded due to early labour activity. In the DHA + AA group, two infants were excluded, one because of preterm birth and one because of maternal gestational diabetes mellitus. The number of mother-infant pairs lost to followup did not differ between the groups $(P=0 \cdot 33)$. Ultimately, 119 mother-infant pairs were evaluated (control, $n 36$; DHA, $n$ 42; DHA + AA, $n$ 41).

\section{Dietary intervention}

All the women received a supplement of vitamins and minerals according to the Dutch RDA. The women were instructed to take two capsules once daily from enrolment until 3 months after delivery. The DHA + AA group received 220 mg DHA (Marinol D40, Lipid Nutrition B.V., Wormerveer, The Netherlands, a DHA-enriched purified fish oil) and $220 \mathrm{mg}$ AA (Wuhan Alking Bioengineering Co. Ltd, Wuhan, China). The DHA group received $220 \mathrm{mg}$ DHA and one capsule containing soyabean oil (Wuhan Alking
Table 1. Daily intakes of fatty acids (in $\mathrm{mg}$ ) from the supplements

\begin{tabular}{lccc}
\hline & Control group & DHA group & DHA + AA group \\
\hline LA & 535 & 274 & 46 \\
ALA & 60 & 32 & 7 \\
AA & 0 & 15 & 220 \\
EPA & 0 & 34 & 36 \\
DHA & 0 & 220 & 220 \\
\hline
\end{tabular}

LA, linoleic acid, 18:2n-6; ALA, $\alpha$-linolenic acid, 18:3n-3; AA, arachidonic acid, $20: 4 n-6 ;$ EPA, $20: 5 n-3 ;$ DHA, $22: 6 n-3$.

Bioengineering Co. Ltd). The control group received two capsules containing soyabean oil. Table 1 shows the daily fatty acid intakes from the capsules for each of the three treatment groups. The daily dosages of AA and DHA were within the range of typical intakes for Western adults ${ }^{(13)}$.

\section{Characteristics}

Prenatal and perinatal data were collected using the obstetrical optimality score form. The form consists of seventy-four variables describing socio-economic status, non-obstetrical conditions during pregnancy, obstetrical history, diagnostic and therapeutic measures, parturition and neonatal condition immediately after birth. The obstetrical optimality score is calculated from the number of items with values within a predefined optimal range ${ }^{(14)}$. Data on infant feeding were collected at 2 and 12 weeks. Infant formula was not provided by the investigators, but since at the time of the study, none of the commercially available formulas contained DHA or AA, and their $\alpha$-linolenic acid (ALA) and linoleic acid (LA) contents were within a narrow range, we consider all formulas equal.

\section{Neurological examination}

The infants' neurological condition was assessed using two instruments. A traditional and detailed neurological assessment $^{(6)}$ was performed 2 weeks after birth (mean $14.7 \mathrm{~d}$ (SD 3.9) d). This examination was used to summarise traditional neurological findings, such as spontaneous motor behaviour, reactions, reflexes and muscle tone regulation. Neurological findings were described in two ways, firstly by using the clinical classification (normal, mildly abnormal and definitely abnormal neurological condition) ${ }^{(15)}$ and secondly by using the neurological optimality score (scored to a maximum of $60)^{(14)}$. Neurological condition was also evaluated on the basis of general movement quality. General movements are movements involving all parts of the body, characterised by variation and complexity. They emerge during early fetal life and disappear at about 4 months post-term when goaldirected movements emerge. The typical characteristics disappear in cases of dysfunction ${ }^{(10)}$. The infants' general movements were assessed twice: in the second postnatal week (the 'writhing' phase) and in the twelfth postnatal week (the 'fidgety' phase). To this end, spontaneous motility in the supine position was videotaped for at least $5 \mathrm{~min}$. General movement quality was assessed according to previously established methods ${ }^{(16)}$. Specifically, movements were classified as normal optimal, normal suboptimal, mildly abnormal or definitely abnormal (Table 2). It is important to emphasise that the label 'mildly abnormal' 
Table 2. Classification of general movement quality and its significance to the current brain condition ${ }^{(7)}$

\begin{tabular}{lllll}
\hline & Complexity & Variation & Fluency & Significance to brain condition \\
\hline Normal optimal GM & +++ & +++ & + & Excellent \\
Normal suboptimal GM & ++ & ++ & - & Typical \\
Mildly abnormal GM & + & + & - & Non-optimal \\
Definitely abnormal GM & - & - & - & Dysfunctional \\
\hline
\end{tabular}

GM, general movements.

is a descriptor of movement quality, indicating lower movement complexity and less variation. In other words, it does not indicate a mildly abnormal brain condition. Mildly abnormal general movements are within the range of "clinically normal' movements, but they reflect a non-optimal condition of the young infant brain. The majority of typically developing infants shows normal suboptimal general movements, and normal optimal general movements are considered as excellent movements. Normal optimal general movements occur in only $10-20 \%$ of 3 -month-old term infants ${ }^{(10,17)}$. Two assessors (S. A. v. G. and M. H.-A.), blinded to the intervention, analysed the videos together. In case of disagreement, the findings were discussed until a consensus was reached.

\section{Fatty acid analysis}

In the 36th week of pregnancy, maternal EDTA-anticoagulated blood was collected by venepuncture. The erythrocyte fatty acid compositions were determined using our previously described capillary GC method with flame ionisation detection $^{(18)}$. The erythrocyte fatty acid compositions are presented in relative amounts, i.e. $\mathrm{g} / 100 \mathrm{~g}$ fatty acids (wt \%) and the DHA/AA ratio in wt/wt.

\section{Statistical analyses}

Statistics were performed with the SPSS software package, version 16 (SPSS Inc., Chicago, IL, USA). The data were evaluated on the basis of intention to treat. The power analysis was based on the neurological optimality score at 2 weeks as the primary outcome parameter. Power analysis revealed that sixty-four infants per group were needed for the detection of a two-point $(=0.5 \mathrm{SD})$ difference in the neurological optimality score (power $=0.85, \alpha=0.05$ ). We were unable to perform a power analysis on general movement quality since the effect of prenatal LCP supplementation on this parameter could not be estimated from any other study. During data collection, we noticed that the number of infants with mildly abnormal general movements at 12 weeks greatly exceeded the numbers found in previous studies with healthy infants (i.e. about $25-30 \%)^{(17,19)}$. The excess proved group specific and subsequent disclosure of blinding revealed that this excess occurred in the DHA-only group. In high-risk infants, the presence of mildly abnormal general movements has been associated with an increase in minor neurological dysfunction at school age ${ }^{(7)}$, although the prognostic value in healthy infants is currently unknown. We decided to discontinue the inclusion of participants. The between-group differences in general movement quality were highly significant, indicating that the group sizes conferred sufficient power to detect subtle differences in neurological development. The identical medians of the neurological optimality score indicated that this parameter exhibited no trend towards between-group differences.

Between-group differences in neurological optimality score, obstetrical optimality score, maternal age, gestational age and birth weight were calculated using a one-way ANOVA with Bonferroni correction at $P<0 \cdot 05$. Between-group differences in general movement scores, percentage of women with higher education, maternal and paternal smoking, parity and sex were calculated using a $\chi^{2}$ test corrected for Bonferroni at $P<0.017 \quad(0.05 / 3$ for three between-group analyses). Linear or logistic multivariate regression was applied to correct the effect of the intervention for potential confounders, such as maternal age, sex, gestational age at birth, birth weight, maternal education, maternal parity, paternal and maternal smoking and duration of breastfeeding. The DHA and DHA + AA groups were compared with the control group in the 'default' model. New models were created excluding the control group to compare differences between the DHA and the DHA + AA groups. A Kruskal-Wallis test was performed to study the effect of supplementation on maternal erythrocyte DHA, AA or DHA/AA; $P<0.017$ was considered to be significant. To study whether maternal erythrocyte DHA, AA or the DHA/AA ratio correlated with the neurological optimality score, a Spearman correlation was used at $P<0 \cdot 05$. Mann-Whitney $U$ tests were used to calculate differences in maternal erythrocyte DHA, AA and DHA/AA for different general movement scores $(P<0 \cdot 05)$.

\section{Ethical statement}

The present study was conducted according to the guidelines laid down in the Declaration of Helsinki, and all procedures involving human subjects were approved by the Central Committee on Research Involving Human Subjects (CCMO, the Hague, The Netherlands; protocol number P03.1071C). All the women provided written informed consent. The trial is registered under ISRCTN58176213.

\section{Results}

The 119 mother-infant pairs did not show any betweengroup differences for the prenatal, perinatal and social characteristics (Table 3).

Maternal erythrocyte fatty acid contents (wt \%) and ratios (wt/wt) in the 36th week of pregnancy were analysed to be used as compliance parameters. Compared with the control group, the erythrocyte DHA was higher after DHA $(P<0.001)$ and DHA + AA $(P<0.001)$ supplementation. Erythrocyte AA was higher in the DHA + AA group than in the DHA group $(P<0 \cdot 001)$. Supplementation of DHA alone 
Table 3. Prenatal, perinatal and social characteristics of the 119 mother-infant pairs included in the study (Mean values and standard deviations or numbers and percentages)

\begin{tabular}{|c|c|c|c|c|c|c|c|}
\hline & \multicolumn{2}{|c|}{$\begin{array}{l}\text { Control group } \\
\quad(n 36)\end{array}$} & \multicolumn{2}{|c|}{$\begin{array}{l}\text { DHA group } \\
\quad(n \text { 42) }\end{array}$} & \multicolumn{2}{|c|}{$\begin{array}{c}\mathrm{DHA}+\mathrm{AA} \\
\text { group }(n 41)\end{array}$} & \multirow[b]{2}{*}{$P$} \\
\hline & Mean & SD & Mean & SD & Mean & SD & \\
\hline Maternal age (years) & $32 \cdot 4$ & $5 \cdot 1$ & 32.5 & 4.4 & $32 \cdot 7$ & $4 \cdot 8$ & 0.96 \\
\hline Gestational age at birth (weeks) & $40 \cdot 2$ & 1.0 & $40 \cdot 2$ & $1 \cdot 1$ & $40 \cdot 2$ & $1 \cdot 1$ & 0.99 \\
\hline Birth weight (g) & 3565 & 547 & 3585 & 462 & 3644 & 373 & 0.74 \\
\hline \multirow[t]{2}{*}{ oos } & $64 \cdot 3$ & 3.8 & 63.8 & 4.0 & 63.4 & 3.8 & 0.64 \\
\hline & $n$ & $\%$ & $n$ & $\%$ & $n$ & $\%$ & $P$ \\
\hline Maternal higher education* & 27 & 77 & 30 & 71 & 27 & 66 & 0.21 \\
\hline Maternal smoking during pregnancy & 2 & 6 & 2 & 5 & 3 & 7 & 0.88 \\
\hline Paternal smoking during pregnancy & 10 & 29 & 9 & 21 & 10 & 24 & 0.72 \\
\hline First born & 21 & 60 & 25 & 60 & 19 & 46 & 0.38 \\
\hline Male sex & 22 & 63 & 16 & 38 & 19 & 46 & 0.091 \\
\hline Exclusively formula fed $t$ & 5 & 14 & 2 & 5 & 6 & 15 & 0.28 \\
\hline Exclusively breastfed after 2 weeks & 29 & 81 & 36 & 86 & 33 & 81 & 0.78 \\
\hline Exclusively formula fed after 12 weeks & 21 & 58 & 28 & 67 & 23 & 56 & 0.59 \\
\hline
\end{tabular}

AA, arachidonic acid; OOS, obstetrical optimality score.

*At least high school completed.

tInfant formula was not provided by the investigators. None of the commercially available formulas contained DHA or AA at the time the study took place.

tended to lower erythrocyte AA $(P=0.085)$. The erythrocyte DHA/AA ratio was highest in the DHA group and lowest in the control group $(P<0.001)$. The DHA + AA group showed an intermediate erythrocyte DHA/AA ratio (DHA + AA group compared with control group, $P=0.004$; DHA + AA group compared with DHA group, $P=0.002$; Table 4). Supplementation also included relatively small amounts of LA, ALA and EPA. Erythrocyte LA and ALA did not differ between the groups. Erythrocyte EPA was higher in mothers in the DHA group compared with the control group $(P=0.004)$ and the $\mathrm{DHA}+\mathrm{AA}$ group $(P=0.009)$. The control and DHA + AA groups did not differ in erythrocyte EPA content $(P=0.635)$.

The distribution of the neonatal neurological classification in the second postnatal week and the general movement quality in the second and twelfth weeks are shown in Table 5. There were no between-group differences in neonatal neurological classification. None of the infants were classified as having a definitely abnormal neonatal neurological condition and the rate of mild abnormality varied between
15 and $20 \%$. The neurological optimality score was identical in the three treatment groups: control, median 52 (range 44-60); DHA, median 52 (46-57); DHA + AA, median 52 (44-58), $P=0.95$. Virtually none of the infants showed normal optimal general movements, and therefore we recoded normal optimal and normal suboptimal general movements into normal general movements. Only one infant displayed definitely abnormal general movements at 2 weeks (control group) and one at 12 weeks (DHA group). We compared normal with mildly abnormal general movements and excluded the infants with definitely abnormal general movements from statistical analyses. Combining these infants with those with mildly abnormal general movements into an abnormal general movements' group did not change the results or conclusions (data not shown). At 2 weeks, infants in the DHA group tended to show mildly abnormal general movements $(54 \%)$ more frequently than the infants in the control group $(36 \%, P=0.13)$ and the DHA + AA group (40\%, $P=0.21$; no difference between control and DHA + AA, $P=0.73$ ). Between-group differences were more pronounced

Table 4. Fatty acids (wt \%) in maternal red blood cells in the 36th week of pregnancy

(Median values with their ranges)

\begin{tabular}{|c|c|c|c|c|c|c|c|}
\hline & \multicolumn{2}{|c|}{ Control group ( $n$ 37) } & \multicolumn{2}{|c|}{ DHA group ( $n 41)$} & \multicolumn{2}{|c|}{$\mathrm{DHA}+\mathrm{AA}$ group $(n 37)$} & \\
\hline & Median & Range & Median & Range & Median & Range & \\
\hline LA & 8.65 & $6.19-10.48$ & 7.95 & $5.92-10.23$ & 8.06 & $5 \cdot 98-13 \cdot 10$ & \\
\hline ALA & 0.19 & $0.08-0.32$ & 0.19 & $0.10-0.34$ & 0.18 & $0.09-0.33$ & \\
\hline AA & $12 \cdot 91$ & $9.95-14.95$ & $12 \cdot 13$ & $9 \cdot 63-15 \cdot 22$ & 13.60 & $11 \cdot 17-15.52$ & c \\
\hline EPA & 0.31 & $0.16-0.56$ & 0.38 & $0 \cdot 19-1 \cdot 31$ & 0.31 & $0.16-0.87$ & $a, c$ \\
\hline DHA & 4.44 & $3.00-6.92$ & $5 \cdot 51$ & $3.98-8 \cdot 20$ & $5 \cdot 57$ & $2.48-8.32$ & $a, b$ \\
\hline DHA/AA & 0.34 & $0.24-0.70$ & 0.46 & $0.34-0.75$ & 0.41 & $0.19-0.69$ & $a, b, c$ \\
\hline
\end{tabular}

LA, linoleic acid; ALA, $\alpha$-linolenic acid; AA, arachidonic acid.

a Significant difference between control group and DHA group.

${ }^{b}$ Significant difference between control group and DHA + AA group.

c Significant difference between DHA and DHA + AA groups; $P<0.017$. 
Table 5. Distribution of neurological classification in the second postnatal week and general movement quality in the second and twelfth postnatal weeks in the three treatment groups

\begin{tabular}{|c|c|c|c|c|c|c|c|c|c|c|c|c|c|c|c|c|}
\hline & \multicolumn{4}{|c|}{ NNC } & & \multicolumn{5}{|c|}{ GM2 } & & \multicolumn{4}{|c|}{ GM12 } & \\
\hline & $\mathrm{N}$ & MA & DA & NA & & $\mathrm{NO}$ & NS & MA & $\mathrm{DA}$ & NA & & $\mathrm{NO}$ & NS & MA & $\mathrm{DA}$ & \\
\hline Control (n 36) & 28 & 7 & 0 & 1 & $a$ & 1 & 19 & 11 & 1 & 4 & $\mathrm{a}$ & 2 & 23 & 11 & 0 & \\
\hline $\mathrm{DHA}(n 42)$ & 35 & 6 & 0 & 1 & $\mathrm{a}$ & 0 & 17 & 20 & 0 & 5 & $\mathrm{a}$ & 0 & 16 & 25 & 1 & b \\
\hline $\mathrm{DHA}+\mathrm{AA}(n 41)$ & 33 & 8 & 0 & 0 & $a$ & 1 & 22 & 15 & 0 & 3 & $\mathrm{a}$ & 1 & 26 & 14 & 0 & $\mathrm{a}$ \\
\hline
\end{tabular}

NNC, neonatal neurological classification at 2 weeks; GM2, general movements at 2 weeks; GM12, general movements at 12 weeks; N, normal; MA, mildly abnormal; DA, definitely abnormal; NO, normal optimal; NS, normal suboptimal; NA, not assessed, as the children were not in the appropriate behavioural state and NNC or GM quality could not be assessed; AA, arachidonic acid.

There were no between-group differences for NNC and GM2.

${ }^{a}$ Identical superscripts indicate that there are no significant differences between treatment groups.

${ }^{b}$ Significantly different from control $(P=0.008)$ and $\mathrm{DHA}+\mathrm{AA}(P=0.015)$.

at 12 weeks: $61 \%$ of the infants in the DHA group showed mildly abnormal general movements compared with $31 \%$ in the control group $(P=0.008)$ and $34 \%$ in the DHA + AA group $(P=0.015 ;$ no difference between control and DHA + AA, $P=0 \cdot 74)$. Multivariate analyses took into account the role of confounders, namely maternal age at inclusion, sex, gestational age at birth, birth weight, maternal education, parity, paternal and maternal smoking and duration of breastfeeding. The neurological optimality scores and neonatal neurological classifications did not differ between the three groups. General movement quality at 2 weeks was significantly lower in the DHA group when compared with the control group. At 12 weeks, infants in the DHA group showed a higher rate of mildly abnormal general movements than those in the control group and DHA + AA group (Table 6).

There were no significant correlations between maternal erythrocyte LA, ALA, AA, EPA, DHA or the DHA/AA ratio and the neurological optimality score. Mann-Whitney $U$ tests revealed that mothers of infants with mildly abnormal general movements at 2 weeks had lower erythrocyte AA (median 12.25; range 9.63-14.92) compared with those of infants with normal general movements (13.03 (9.95-15.52), $P=0.022$ ). General movement quality at 12 weeks did not relate to maternal erythrocyte LA, ALA, AA, EPA, DHA or the DHA/AA ratio.

\section{Discussion}

The present study indicates that supplementation with DHA alone, but not DHA + AA, is associated with a higher rate of mildly abnormal general movements. The traditional neurological examination did not reveal between-group differences in neonatal neurological classification or neurological optimality scores. We realise that the study lacks the power to permit the conclusion that supplementation with DHA alone or with DHA + AA does not affect neurological optimality scores. However, the finding that the median values of the neurological optimality score were identical in the three groups suggests that increasing the number of participants would not lead to a different conclusion.

Table 6. Effects of intervention after correction for potential confounders

\begin{tabular}{|c|c|c|c|c|}
\hline & Coefficient B & $95 \% \mathrm{Cl}$ & $P$ & Explained variance $r^{2 *}$ \\
\hline NOS & & & & $8.6 \%$ \\
\hline Control v. DHA & 0.022 & $-1.266,1.534$ & 0.85 & \\
\hline Control v. DHA + AA & 0.003 & $-1.398,1.440$ & 0.98 & \\
\hline $\mathrm{DHA} v . \mathrm{DHA}+\mathrm{AA}$ & $\begin{array}{c}-0.012 \\
\operatorname{Exp~B}\end{array}$ & $\begin{array}{l}-1 \cdot 454,1.315 \\
95 \% \mathrm{Cl}\end{array}$ & $\begin{array}{c}0.92 \\
P\end{array}$ & Nagelkerke $r^{2}(\%) \cdot \chi^{2} \cdot P^{*}$ \\
\hline MA NNC & & & & $27.5 ; 21.0 ; 0.050$ \\
\hline Control v. DHA & 0.814 & $0.208,3.184$ & 0.77 & \\
\hline Control v. $\mathrm{DHA}+\mathrm{AA}$ & $1 \cdot 141$ & $0.295,4.409$ & 0.85 & \\
\hline $\mathrm{DHA} v \cdot \mathrm{DHA}+\mathrm{AA}$ & 1.589 & $0.427,5.908$ & 0.49 & \\
\hline MA GM at 2 weeks & & & & $14.2 ; 11.3 ; 0.51$ \\
\hline Control v. DHA & 3.867 & $1 \cdot 228,12 \cdot 173$ & 0.021 & \\
\hline Control v. DHA + AA & 1.854 & $0.585,5.880$ & 0.29 & \\
\hline $\mathrm{DHA} v \cdot \mathrm{DHA}+\mathrm{AA}$ & 0.511 & $0.188,1.390$ & 0.19 & \\
\hline MA GM at 12 weeks & & & & $30 \cdot 4 ; 28 \cdot 7 ; 0.004$ \\
\hline Control v. DHA & $4 \cdot 121$ & $1 \cdot 335,12 \cdot 719$ & 0.014 & \\
\hline Control v. DHA + AA & $1 \cdot 162$ & $0.366,3.686$ & $0 \cdot 80$ & \\
\hline $\mathrm{DHA} v . \mathrm{DHA}+\mathrm{AA}$ & 0.264 & $0.089,0.788$ & 0.017 & \\
\hline
\end{tabular}

NOS, neurological optimality score; AA, arachidonic acid; MA, mildly abnormal; NNC, neonatal neurological classification; GM, general movements.

* The explained variance is assessed in the models in which both the DHA and DHA + AA groups are compared with the control group. To compare the DHA group with the DHA + AA group, a new model was created from which the control group was excluded. The first mentioned group functions as the reference group (i.e. $=0$ ). The effects of the intervention are corrected for maternal age (at inclusion), sex, gestational age at birth, birth weight, maternal education, parity, paternal and maternal smoking and duration of breastfeeding. 
We used general movement quality as a parameter for neurodevelopment, since it proved sensitive to postnatal LCP supplementation in term infants in a previous study ${ }^{(19)}$. In the present study, we found more mildly abnormal general movements after supplementation of DHA alone, whereas general movement quality after supplementation of $\mathrm{DHA}+\mathrm{AA}$ was comparable to that in the control group. The latter suggests that the addition of AA neutralised the effect of DHA. DHA + AA did not influence general movement quality compared with the control group. The label mildly abnormal is a descriptor of movement quality, indicating lower movement complexity and less variation (Table 2). It indicates non-optimal rather than mildly abnormal current brain condition. Although there is no information on lowrisk populations, the presence of mildly abnormal general movements in high-risk populations is associated with an increased prevalence of minor neurological dysfunction and behavioural problems at school age. The presence of mildly abnormal general movements, however, cannot be used as a single predictor of later outcome. Nevertheless, accumulating evidence indicates that the degree of variability in motor behaviour is a marker of the functional integrity of the developing nervous system, in particular of the integrity of cerebral connectivity, i.e. white matter function ${ }^{(10,20)}$.

Generally, DHA status is positively associated with neurological development. We supplemented a relatively low dose of DHA. Maternal DHA supplementation studies during pregnancy or during pregnancy and subsequent lactation with doses ranging from 200 to $3300 \mathrm{mg}$ DHA/d resulted in improved visual acuity at 4 months ${ }^{(21)}$, enhanced problem solving at 9 months ${ }^{(22)}$, improved eye and hand coordination at 2.5 years $^{(23)}$ and a higher IQ at 4 years $^{(24)}$, whereas other developmental parameters showed no benefit ${ }^{(4)}$. Since the reported beneficial effects of DHA do not seem dose dependent or duration dependent (supplementation during pregnancy or during pregnancy and subsequent lactation) and the effects are mainly found in older infants and toddlers, it is conceivable that the positive effects of DHA on cognition first become expressed after early infancy. Furthermore, the relationship between DHA intake and neurological development may be non-linear, for which there is some evidence from animal studies ${ }^{(25,26,27)}$. Negative associations between fish intake during pregnancy and fetal growth ${ }^{(28,29,30)}$ have been shown.

Bouwstra et al. ${ }^{(9)}$ reported that general movement quality is associated with prenatal AA status rather than prenatal DHA status. In the present study, the effect of supplemental DHA was neutralised by additional AA, while supplementation of DHA alone tended to lower maternal AA status. These observations could indicate that the effect of DHA supplementation on general movement quality is related to the lower maternal AA status.

The average daily intakes of DHA and AA in The Netherlands are estimated to amount to $100 \mathrm{mg} / \mathrm{d}$ each. Accordingly, he average fish intake in the present study population amounts to 0.94 meals per week, of which 0.45 meals include fatty fish $^{(11)}$. Consequently, supplementation of $220 \mathrm{mg}$ DHA increased the dietary DHA/AA ratio, while this ratio became largely restored by the additional supplementation of $220 \mathrm{mg}$ $\mathrm{AA}$ in the DHA + AA group. The similarities between the dietary DHA/AA ratios of the control and $\mathrm{DHA}+\mathrm{AA}$ groups were reflected by a similarly low percentage of mildly abnormal general movements, while the higher dietary DHA/AA ratio of the DHA group proved causally related to a substantially increased occurrence of mildly abnormal general movements (Table 4). The estimated dietary DHA/AA ratios of the three groups were, however, not fully reflected by the corresponding erythrocyte DHA/AA ratios, since here the DHA + AA group occupied a position intermediate to the control and DHA groups (Table 6). This apparent discrepancy between the dietary and erythrocyte DHA/AA ratios suggests that it is the maternal dietary DHA/AA ratio rather than the widely employed erythrocyte DHA/AA status, which is implicated in the occurrence of mildly abnormal general movements.

Various brain regions in neonatal baboons differ in DHA, $\mathrm{AA}$ and consequently the DHA/AA ratio ${ }^{(31)}$. This may imply region-specific regulation of brain LCP contents, for instance by fatty acid-binding proteins ${ }^{(32)}$ and other as yet poorly understood mechanisms. Grey matter contains high amounts of DHA and AA in contrast to white matter ${ }^{(31)}$. It can be surmised that white matter LCP contents may be less well regulated and consequently be more sensitive to DHA or AA changes than other brain regions. Since DHA and AA are known to compete for incorporation in the brain $^{(33,34)}$, supplementation of DHA may lead to a decrease in AA, especially in white matter, whereas additional AA supplementation restores the DHA/AA balance. In addition, white matter shows a low DHA/AA ratio, i.e. more AA than DHA, when it is compared with grey matter. Since general movement quality is associated with the integrity of the white matter ${ }^{(10,20)}$, this could explain why general movement quality is sensitive to the dietary DHA/AA ratio.

DHA depletion is undoubtedly associated with altered brain function $^{(27)}$. Although the current Western diet is considered to be at least marginal in DHA, results from DHA supplementation studies remain inconclusive ${ }^{(2,35)}$. We hypothesise that various brain regions differ in LCP contents due to differences in regulation. It should be realised that different neurodevelopmental tests subserve the evaluation of different brain functions. In addition, it is likely that brain regions and functions exhibit different sensitivities to changes in LCP status. For example, Dunstan et al. ${ }^{(23)}$ showed a beneficial effect on eye and hand coordination, but not on other subscales of the Griffith Mental Developmental Scales, the Peabody Picture Vocabulary Test, or the Child Behaviour Checklist. Maternal fish oil supplementation during 4 months of lactation did not affect problem-solving ability at 9 months of age, but at 1 year and 2 years, the fish oil group performed less well on the MacArthur Communicative Developmental Inventory, especially the boys ${ }^{(36)}$. The various risk factors exhibit different dose responses to LCP $n-3$ in CVD as well ${ }^{(37)}$. Differences in sensitivity may in part explain why the use of various tests at various ages led to different results and conclusions regarding the effects of LCP supplementation on developmental outcome.

We conclude that general movement quality is sensitive to maternal dietary DHA/AA balance during pregnancy and lactation. Supplementation of DHA without AA caused an increase in mildly abnormal general movements in early infancy, which in high-risk infants has been associated with a non-optimal brain condition. However, the prognostic 
significance of a non-optimal brain condition in healthy term infants is currently unknown. Therefore, our data stress the need for a long-term follow-up with sensitive and functionspecific instruments in studies evaluating the effect of early LCP supplementation on neurodevelopmental outcome.

\section{Acknowledgements}

D. A. J. D.-B., A. S. and F. A. J. M. designed the study. S. A. v. G. and B. D. collected the samples and were involved in writing the paper. S. A. v. G. and M. H.-A. assessed general movement quality. S. A. v. G., D. A. J. D.-B., M. H.-A., J. J. H. M. E and F. A. J. M. analysed the results and wrote the report. None of the authors had any conflict of interest. We kindly thank Remko S. Kuipers, Ilona B.M. Meijer and Ingrid A. Martini for their valuable aid in the analysis of the erythrocyte fatty acids. The present study was financially supported by FrieslandCampina, The Netherlands.

\section{References}

1. Innis SM (2007) Fatty acids and early human development. Early Hum Dev 83, 761-766.

2. McCann JC \& Ames BN (2005) Is docosahexaenoic acid, an $n-3$ long-chain polyunsaturated fatty acid, required for development of normal brain function? An overview of evidence from cognitive and behavioral tests in humans and animals. Am J Clin Nutr 82, 281-295.

3. Clandinin MT, Chappell JE, Leong S, et al. (1980) Intrauterine fatty acid accretion rates in human brain: implications for fatty acid requirements. Early Hum Dev 4, 121-129.

4. Hadders-Algra M (2008) Prenatal long-chain polyunsaturated fatty acid status: the importance of a balanced intake of docosahexaenoic acid and arachidonic acid. J Perinat Med 36, $101-109$.

5. Al MD, Van Houwelingen AC, Kester AD, et al. (1995) Maternal essential fatty acid patterns during normal pregnancy and their relationship to the neonatal essential fatty acid status. Br J Nutr 74, 55-68.

6. Prechtl H (1977) The neurological examination of the full-term newborn infant. Clinics in Developmental Medicine No. 63, 2nd ed.

7. Hadders-Algra M (2004) General movements: a window for early identification of children at high risk for developmental disorders. J Pediatr 145, S12-S18.

8. Dijck-Brouwer DA, Hadders-Algra M, Bouwstra $\mathrm{H}$, et al. (2005) Lower fetal status of docosahexaenoic acid, arachidonic acid and essential fatty acids is associated with less favorable neonatal neurological condition. Prostaglandins Leukot Essent Fatty Acids 72, 21-28.

9. Bouwstra H, Dijck-Brouwer DA, Decsi T, et al. (2006) Relationship between umbilical cord essential fatty acid content and the quality of general movements of healthy term infants at 3 months. Pediatr Res 59, 717-722.

10. Hadders-Algra M (2007) Putative neural substrate of normal and abnormal general movements. Neurosci Biobehav Rev 31, $1181-1190$

11. Doornbos B, van Goor SA, Dijck-Brouwer DA, et al. (2009) Supplementation of a low dose of DHA or DHA + AA does not prevent peripartum depressive symptoms in a small population based sample. Prog Neuropsychopharmacol Biol Psychiatry 33, 49-52.
12. van Goor SA, Dijck-Brouwer DA, Hadders-Algra M, et al. (2009) Human milk arachidonic acid and docosahexaenoic acid contents increase following supplementation during pregnancy and lactation. Prostaglandins Leukot Essent Fatty Acids 80, 65-69.

13. Astorg P, Arnault N, Czernichow S, et al. (2004) Dietary intakes and food sources of $n-6$ and $n-3$ PUFA in French adult men and women. Lipids 39, 527-535.

14. Touwen BC, Huisjes HJ, Jurgens-van der Zee AD, et al. (1980) Obstetrical condition and neonatal neurological morbidity. An analysis with the help of the optimality concept. Early Hum Dev 4, 207-228.

15. Jurgens-van der Zee AD, Bierman-van Eendenburg ME, Fidler VJ, et al. (1979) Preterm birth, growth retardation and acidemia in relation to neurological abnormality of the newborn. Early Hum Dev 3, 141-154.

16. Hadders-Algra M, Mavinkurve-Groothuis AM, Groen SE, et al. (2004) Quality of general movements and the development of minor neurological dysfunction at toddler and school age. Clin Rehabil 18, 287-299.

17. Bouwstra H, Dijk-Stigter GR, Grooten HM, et al. (2009) Prevalence of abnormal general movements in three-monthold infants. Early Hum Dev 85, 399-403.

18. Muskiet FA, van Doormaal JJ, Martini IA, et al. (1983) Capillary gas chromatographic profiling of total long-chain fatty acids and cholesterol in biological materials. J Chromatogr 278, 231-244.

19. Bouwstra H, Dijck-Brouwer DA, Wildeman JA, et al. (2003) Long-chain polyunsaturated fatty acids have a positive effect on the quality of general movements of healthy term infants. Am J Clin Nutr 78, 313-318.

20. Spittle AJ, Brown NC, Doyle LW, et al. (2008) Quality of general movements is related to white matter pathology in very preterm infants. Pediatrics 121, e1184-e1189.

21. Judge MP, Harel O \& Lammi-Keefe CJ (2007) A docosahexaenoic acid-functional food during pregnancy benefits infant visual acuity at four but not six months of age. Lipids $\mathbf{4 2}$, $117-122$.

22. Judge MP, Harel O \& Lammi-Keefe CJ (2007) Maternal consumption of a docosahexaenoic acid-containing functional food during pregnancy: benefit for infant performance on problem-solving but not on recognition memory tasks at age 9 mo. Am J Clin Nutr 85, 1572-1577.

23. Dunstan JA, Simmer K, Dixon G, et al. (2006) Cognitive assessment at 21/2 years following fish oil supplementation in pregnancy: a randomized controlled trial. Arch Dis Child Fetal Neonatal Ed 93, F45-F50.

24. Helland IB, Smith L, Saarem K, et al. (2003) Maternal supplementation with very-long-chain n-3 fatty acids during pregnancy and lactation augments children's IQ at 4 years of age. Pediatrics 111, e39-e44.

25. Wainwright PE, Jalali E, Mutsaers LM, et al. (1999) An imbalance of dietary essential fatty acids retards behavioral development in mice. Physiol Behav 66, 833-839.

26. Levant B, Ozias MK \& Carlson SE (2006) Sex-specific effects of brain LC-PUFA composition on locomotor activity in rats. Physiol Behav 89, 196-204.

27. Church MW, Jen KL, Dowhan LM, et al. (2008) Excess and deficient omega-3 fatty acid during pregnancy and lactation cause impaired neural transmission in rat pups. Neurotoxicol Teratol 30, 107-117.

28. Grandjean P, Bjerve KS, Weihe P, et al. (2001) Birthweight in a fishing community: significance of essential fatty acids and marine food contaminants. Int J Epidemiol 30, 1272-1278.

29. Oken E, Kleinman KP, Olsen SF, et al. (2004) Associations of seafood and elongated $n-3$ fatty acid intake with fetal growth and length of gestation: results from a US pregnancy cohort. Am J Epidemiol 160, 774-783. 
30. Thorsdottir I, Birgisdottir BE, Halldorsdottir S, et al. (2004) Association of fish and fish liver oil intake in pregnancy with infant size at birth among women of normal weight before pregnancy in a fishing community. Am $J$ Epidemiol 160, 460-465.

31. Diau GY, Hsieh AT, Sarkadi-Nagy EA, et al. (2005) The influence of long chain polyunsaturate supplementation on docosahexaenoic acid and arachidonic acid in baboon neonate central nervous system. BMC Med 3, 11 .

32. Owada Y (2008) Fatty acid binding protein: localization and functional significance in the brain. Tohoku J Exp Med 214, $213-220$.

33. Simopoulos AP (1991) Omega-3 fatty acids in health and disease and in growth and development. Am J Clin Nutr 54, $438-463$.
34. van Goor SA, Dijck-Brouwer DA, Fokkema MR, et al. (2008) Maternal and fetal brain contents of docosahexaenoic acid (DHA) and arachidonic acid (AA) at various essential fatty acid (EFA), DHA and AA dietary intakes during pregnancy in mice. Prostaglandins Leukot Essent Fatty Acids 78, 159-169.

35. Simmer K, Patole SK \& Rao SC (2008) Longchain polyunsaturated fatty acid supplementation in infants born at term. The Cochrane Database of Systematic Reviews, CD000376.

36. Lauritzen L, Jorgensen MH, Olsen SF, et al. (2005) Maternal fish oil supplementation in lactation: effect on developmental outcome in breast-fed infants. Reprod Nutr Dev 45, 535-547.

37. Mozaffarian D \& Rimm EB (2006) Fish intake, contaminants, and human health: evaluating the risks and the benefits. JAMA 296, 1885-1899. 